\title{
Element-resolved Kikuchi pattern measurements of non-centrosymmetric materials
}

\author{
Maarten $\operatorname{Vos}^{\mathrm{a}}$, Aimo Winkelmann ${ }^{\mathrm{b}}$ \\ ${ }^{a}$ Electronic Materials Engineering Department, Research School of Physics and \\ Engineering, The Australian National University, Canberra, Australia 2601 \\ ${ }^{b}$ Bruker Nano GmbH, Am Studio 2D, 12489 Berlin, Germany
}

\begin{abstract}
Angle-resolved electron Rutherford backscattering (ERBS) measurements using an electrostatic electron energy analyser can provide unique access to elementresolved crystallographic information. We present Kikuchi patterns measurements of the non-centrosymmetric crystal GaP, separately resolving the contributions of electrons backscattered from Ga and P. In comparison to elementintegrated measurements like in the method of electron backscatter diffraction (EBSD), the effect of the absence of a proper 4-fold rotation axis in the point group of $\mathrm{GaP}$ can be sensed with a much higher visibility via the elementresolved Ga to $\mathrm{P}$ intensity ratio. These element-resolved measurements make it possible to experimentally attribute the previously observed point-group dependent effect in element-integrated EBSD measurements to the larger contribution of electrons scattered from Ga compared to P.
\end{abstract}

Keywords: Electron backscatter diffraction;electrostatic analyser; non-centro symmetric crystals; electron Rutherford backscattering; gallium phosphide;

\section{Introduction}

In contrast to the elemental group IV cubic semiconductors, the closely related III-V compound semiconductors lack inversion symmetry. As a consequence, the $\langle 001\rangle$ axes lack a proper 4-fold rotational symmetry. Instead, only an improper 4-fold roto-inversion axis is present, and the point group of the cubic crystals is $\overline{4} 3 \mathrm{~m}$. Determination of the absolute orientation of such a crystal is not only a classic problem in crystallography [1], but it is also a technologically relevant question for the controlled growth of optoelectronic materials like $\mathrm{GaP}$ or GaN on different substrates [2, 3, 4, 5, 6, 7, 8. Electron diffraction experiments are, due to their strong interaction, sensitive to the lack of inversion symmetry in crystals. Recently, it was demonstrated how Electron Backscatter Diffraction (EBSD) can determine the polarity of crystallites in poly-crystalline

Email address: maarten.vos@anu.edu.au (Maarten Vos) 
samples, in particular due to the asymmetry of the shape of the $\{111\}$ Kikuchi bands [9]. However, calculations suggested that also near the $\langle 111\rangle$ zone axes there should be an influence of the polarity of the crystal on the measured EBSD distribution 10. EBSD has also been used to probe the lack of inversion symmetry in ferroelectrics [1].

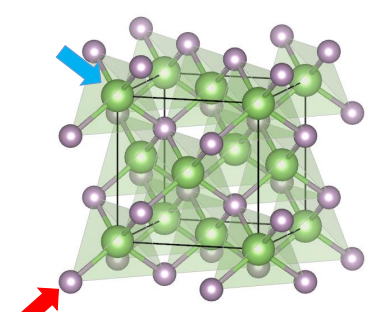

(a)

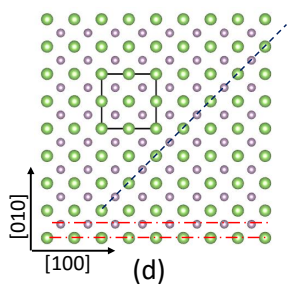

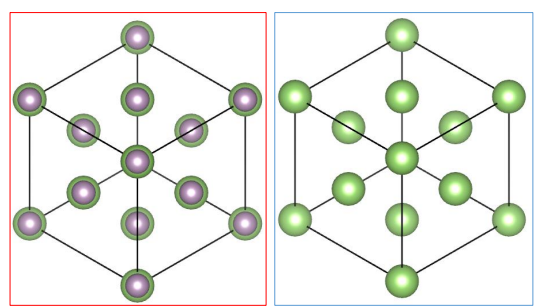

(b)

(c)

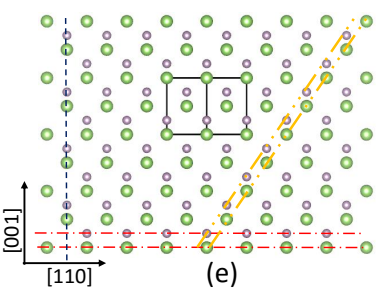

Figure 1: In (a) the conventional unit cell of GaP is shown. The unit cell can be projected along $\langle 111\rangle$ in two ways: For the direction indicated by the red arrow in (a) the $\mathrm{P}$ atoms appear just in front the $\mathrm{Ga}$ atoms (b) along the blue arrow the $\mathrm{P}$ atoms are just behind the $\mathrm{Ga}$ atoms (c). The $\left\{\begin{array}{lll}1 & 0 & 0\end{array}\right\}$ planes (red, dashed-dotted line) and $\left\{\begin{array}{lll}1 & 1 & 0\end{array}\right\}$ planes (blue dashed line) are shown in (d-e). The $\left\{\begin{array}{lll}1 & 0 & 0\end{array}\right\}$ planes are symmetrically spaced and contain either exclusively $\mathrm{Ga}$ or $\mathrm{P}$ atoms. The $\left\{\begin{array}{lll}1 & 1 & 0\end{array}\right.$ planes contain both $\mathrm{Ga}$ and $\mathrm{P}$. The $\{111\}$ planes (yellow line in (e)) contain either Ga or P atoms, but in contrast to the $\left\{\begin{array}{ll}10 & 0\end{array}\right\}$ planes, the Ga plane is not positioned symmetrically between the $\mathrm{P}$ planes. The Kikuchi intensity related to the $\{111\}$ planes is expected to be asymmetric due to the non-centrosymmetric layering of the atoms.

In conventional EBSD, the measurement integrates the signal of all electrons scattered from different nuclei in a compound. However, using an electrostatic analyser with sufficient energy resolution $(0.4 \mathrm{eV})$ at relatively high electron energies $(40 \mathrm{keV})$, it is possible to discriminate electrons scattered from light and heavy elements based on the difference in recoil energy that is transferred by the scattering electron to a target atom (electron Rutherford backscattering, ERBS [12]). This sensitivity to the nuclear mass of the backscattering atoms makes it possible to study separately the individual Kikuchi patterns for electrons scattered from light and heavy elements. In our initial observations, only the intensity distribution along a line of scattering directions could be obtained in this way [13. Recently, this technique was automated so that we can measure extended two-dimensional intensity distributions of Kikuchi patterns, which strongly facilitates the comparison with theoretical simulations [14].

In this paper, we present element-resolved, two-dimensional Kikuchi patterns for the case of cubic GaP, which serves as a prototypical non-centrosymmetric III-V compound crystal. By investigating the diffraction patterns of electrons backscattered incoherently from $\mathrm{Ga}$ and $\mathrm{P}$ separately, we aim to get a better 
understanding of the origin of the sensitivity in EBSD to the polarity of crystal lattice planes and the absolute orientation of non-centrosymmetric crystals. Our experiments are also closely related to photoelectron diffraction [15, 16, 17, where element sensitivity is based on the binding energy levels of the photoexcited core electrons rather than nuclear mass dependent recoil losses in our case. As the energies used for ERBS are considerably higher than even in hard X-ray photoelectron diffraction, the measured intensity distributions potentially contain more detail due to the bulk crystal structure and are thus inherently less surface-sensitive.

So far we have used element-resolved Kikuchi patterns to study the effect of the atomic arrangements in the physical planes of crystals. For GaP, the $\left\{\begin{array}{lll}0 & 1 & 1\end{array}\right\}$ planes contain either Ga or P atoms (Fig. 1(d,e )) with the P-containing planes exactly in between the Ga-containing planes. It turns out that under these conditions the Kikuchi band of the lighter atom has a minimum in intensity centered at this plane, whereas the heavier has a maximum [18. For $\{110\}$ planes $\mathrm{Ga}$ and $\mathrm{P}$ atoms line up in the same plane (Fig. 1(d,e )). It was demonstrated that under these conditions the Kikuchi bands of the two different atoms are virtually indistinguishable [18, 19]

In Fig. 1 we show the GaP crystal and highlight the fact that the two $\langle 111\rangle$ directions of the point lattice marked by a blue and red arrow are not equivalent for the crystal structure occupying the lattice, i.e. we have to distinguish between two inequivalent sets of $\langle 111\rangle$ and $\langle\overline{1} 11\rangle$ directions. Also, the $\{111\}$ lattice planes are of particular interest as they are affected by the absence of the inversion symmetry. Ga and $\mathrm{P}$ atoms are here in different planes, but the $\mathrm{P}$ plane is only slightly displaced from the Ga plane. Now there are two possibilities: the Ga plane just above or just below the $\mathrm{P}$ plane. The element-integrated Kikuchi band shows an asymmetry here 9, 10 reflecting the asymmetry of this alignment. In this paper, we will investigate how this asymmetry manifests itself when one measures the Kikuchi band of $\mathrm{Ga}$ and $\mathrm{P}$ separately.

The quantitative description of Kikuchi bands is possible by the dynamical theory of electron diffraction, e.g. based on a Bloch wave approach. Especially at lower energies (e.g. in the context of photo-electron diffraction) the results can also be qualitatively described by forward scattering of electrons along strings of atoms. Within this framework the $\langle 111\rangle$ directions are expected to depend on the polarity of the crystal planes. Along these directions, the $\mathrm{P}$ atom is either just before or just after the Ga atom, and this sequence is repeated at a distance considerable larger than the Ga-P separation. In the scope of this forward scattering model, we can expect large effects when the intensity in the $\langle 111\rangle$ directions is compared with the intensity in the $\langle\overline{1} 11\rangle$ directions.

In this paper, we demonstrate the unique access to element resolved crystallographic orientation information that is provided by angle-resolved ERBS measurements. We will focus our study mainly on directions along the (110) and ( 110$)$ Kikuchi bands. The (110) and (110) bands themselves are not sensitive to rotation over $90^{\circ}$ but they intersect with the directions of interest: $\langle\overline{1} 11\rangle /\langle\overline{1} \overline{1} 1\rangle$, the $\{111\}$ planes at the $\langle\overline{1} \overline{1} 2\rangle /\langle\overline{1} 12\rangle$ zone axes as well as the $\left[\begin{array}{lll}0 & 0 & 1\end{array}\right]$ zone axis. 

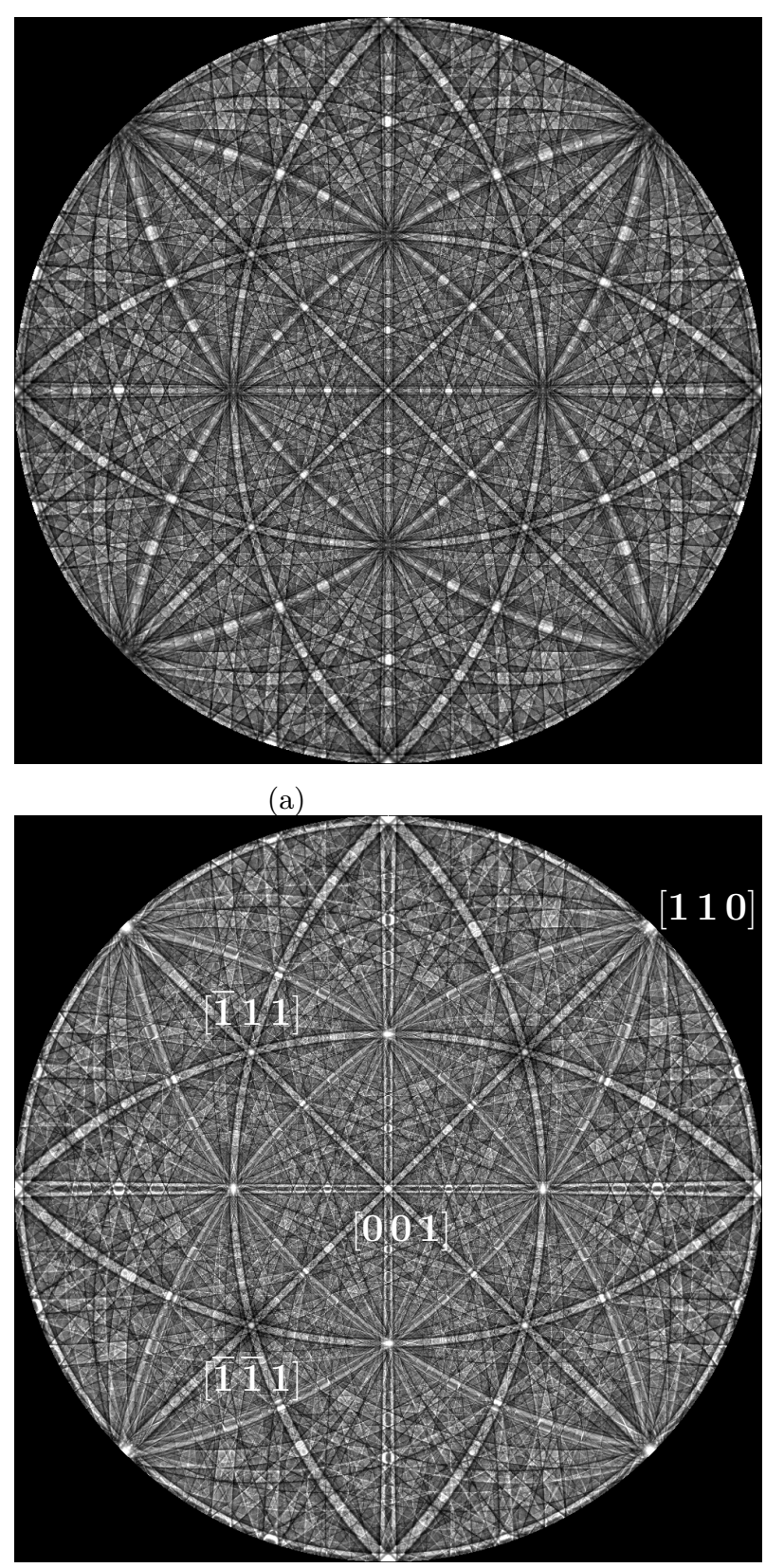

(b)

Figure 2: Element-resolved Kikuchi signals for GaP. Stereographic projection centered on $\left[\begin{array}{lll}0 & 0 & 1\end{array}\right]$. (a) Ga, (b) $\mathrm{P} \times 4$ 


\section{Theory}

The Kikuchi patterns for GaP were simulated using the Bloch wave approach of dynamical electron diffraction theory [20. The relative backscattering strength of $\mathrm{Ga}$ and $\mathrm{P}$ were assumed to scale like the square of the atomic charges $Z^{2}$, as given by the Rutherford scattering cross section. For the actual calculations, we used the software ESPRIT DynamicS (Bruker Nano, Berlin). For GaP about 2200 reflectors $h k l$ with a maximum reciprocal lattice point distance of $1 / d_{h k l}>1 / 0.035 \mathrm{~nm}^{-1}$ and a relative strength of larger than $8 \%$ of the strongest reflector intensity (square of the absolute value of the structure factor) have been taken into account. A mean square displacement of $0.00013 \mathrm{~nm}^{2}$ (both for Ga and P) was used to consider thermal vibrations of the crystal structure via the Debye-Waller factor. To describe the effect of inelastic scattering, an inelastic mean free path of $26 \mathrm{~nm}$ was assumed.

The resulting Kikuchi patterns are shown in a stereographic projection in Fig. 2 for the contribution of Ga and P separately. The $\{100\}$ planes are horizontal and vertical, the $\{110\}$ planes are at $45^{\circ}$ with this. The $\{111\}$ bands in this projection are curved so that they intersect the $\{110\}$ bands in the $x y$-plane at the [1 10$]$-type directions at the edge of the projected hemisphere.

The calculated stereographic projection of the conventional EBSD Kikuchi pattern intensity, i.e. integrated over $\mathrm{Ga}$ and $\mathrm{P}$, is shown in Fig.3a. As our experiment separates these contributions, it is also of interest to plot the Ga to $\mathrm{P}$ intensity ratio. This is done in Fig. 3b. For the center of the $\{100\}$ planes the Ga intensity ratio is enhanced over that of $\mathrm{P}$ (horizontal and vertical line through the center). The $\{110\}$-type planes are clearly visible in the Kikuchi pattern (Fig. 3a but are totally absent in the plot of the Ga to $\mathrm{P}$ ratio figure (Fig. 3b). This implies that $\{110\}$-type planes have exactly the same shape for electrons scattered from Ga and P.

It is of particular interest to look at the shape of the Ga-to-P ratio plot for the $\{111\}$-type planes in figure $3 \mathrm{~b}$. Here a high Ga-to-P ratio (white) band is adjacent to a low Ga-to-P ratio (black) band. For the $\{111\}$ bands that connect 'north-east' to 'south-west' the black band is at the inside of the white band. For those that connect 'north-west' to 'south-east' it is at the outside.

Either the total Kikuchi pattern or the Ga-to-P intensity ratio can be rotated over $90^{\circ}$, but the resulting pattern is slightly different due to the absence of the 4-fold symmetry. To illustrate this we plot $\left.\left(I_{0}-I_{90}\right)\right) /\left(I_{0}+I_{90}\right)$ ( with $I_{0}$ and $I_{90}$ as the intensity before and after rotation, respectively) for both the total Kikuchi pattern (Fig. 3c) and the Ga-to-P ratio (Fig. 3d). The Ga-to-P ratio is much more sensitive to the rotation than the total intensity. Such a behavior indicates that when the Ga intensity increases by rotation over $90^{\circ}$ the $\mathrm{P}$ intensity decreases. Hence the effect of rotation is partly canceled in the plot of the sum, but enhanced in the plot of their ratio.

Besides the $\{111\}$-type planes also the [111] -zone axes display a very peculiar behavior. There are no planes visible intersecting at these directions in the ratio plot, but still there is generally an enhancement of the ratio visible for two and a decline for the other two. 


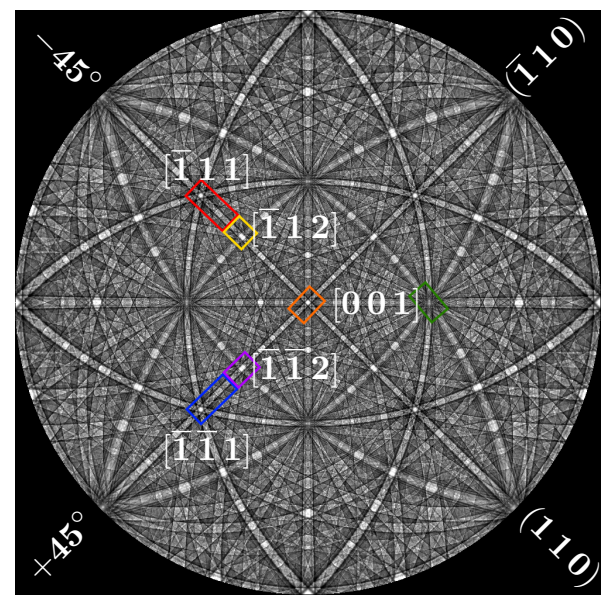

(a)

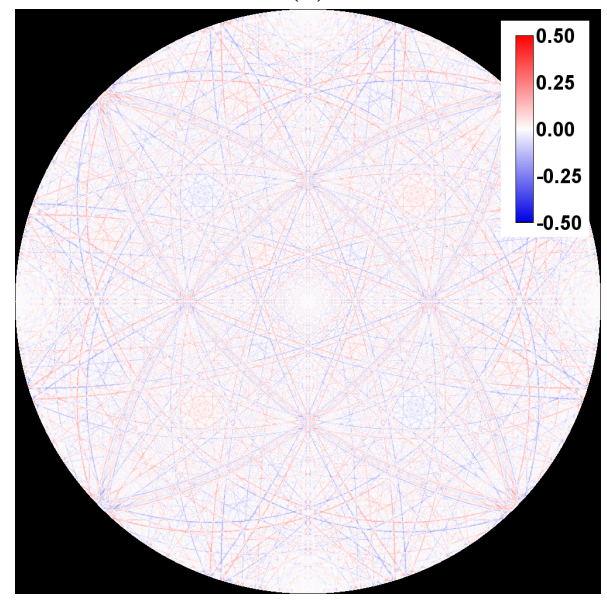

(c)

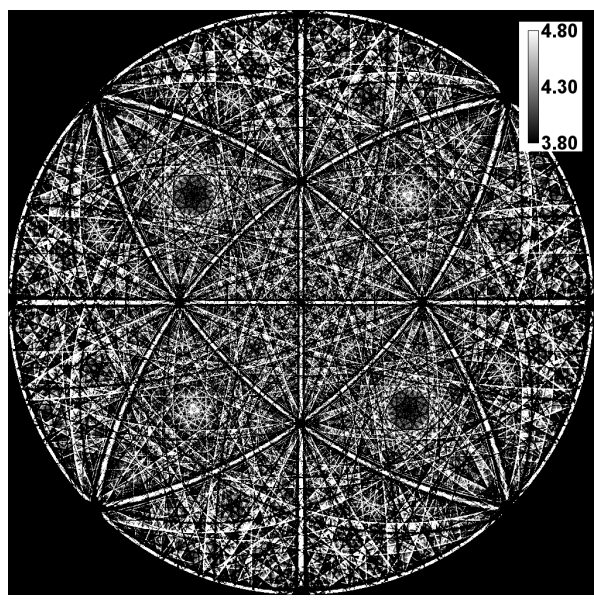

(b)

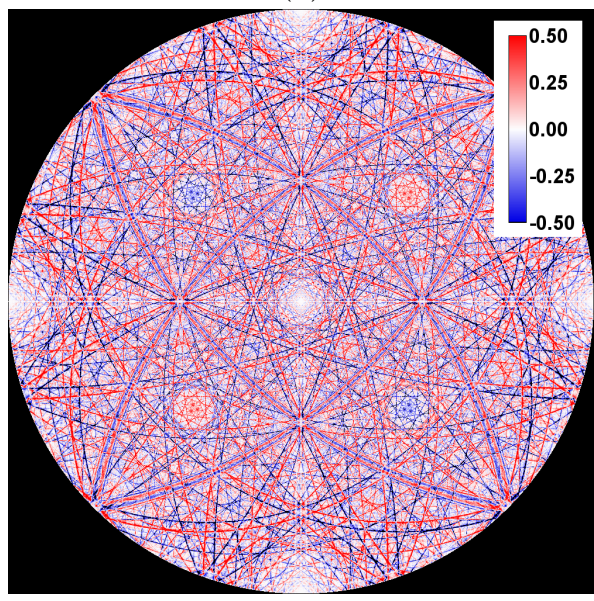

(d)

Figure 3: Simulation of element-integrated (left column) and element-resolved (right column) Kikuchi signals for GaP. Stereographic projection centered on [001]. Top row: (a) elementintegrated Kikuchi intensity of $\mathrm{Ga}+\mathrm{P}$, (b) element-resolved ratio of intensity backscattered by Ga relative to $\mathrm{P}$. The average value of the intensity ratio should be near $Z_{G a}^{2} / Z_{P}^{2}=4.3$. Bottom row: (c) relative asymmetry of (a) after a $90^{\circ}$ rotation around $[001]$. A maximum effect of up to $10 \%$ is seen for the $\{111\}$-type lattice planes. (d) relative asymmetry of (b), with maximum asymmetry near $80 \%$. For both types of measurements, only specific bands are sensitive to the absence of the 4 -fold rotation axis. Most notably, the $\{100\}$ and $\{110\}$ lattice planes show a 4 -fold symmetry indicated by a difference value near zero. 


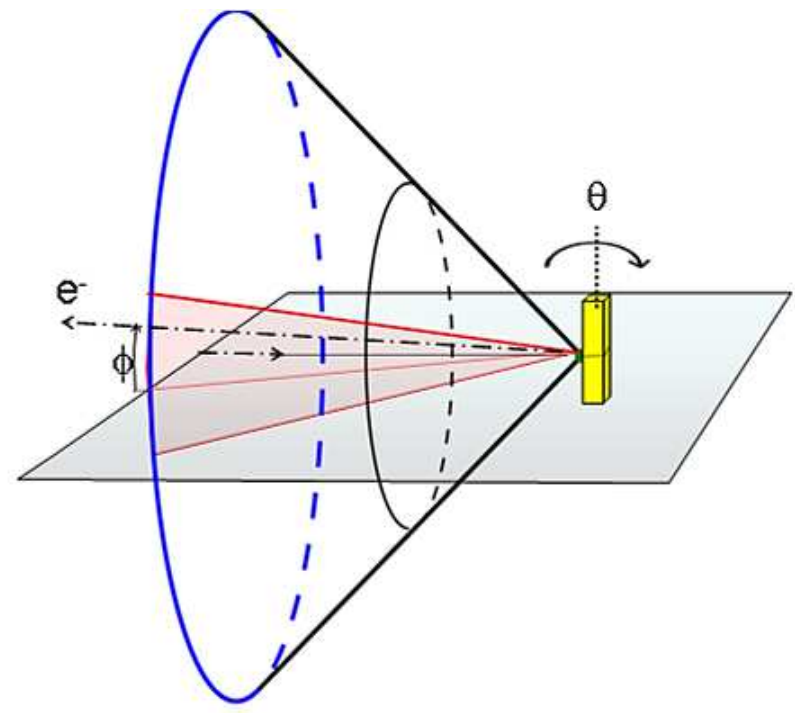

Figure 4: A schematic of the experiment. The spectrometer measures electrons that move very close to a section of the surface of a cone, and resolves their $\phi$ coordinate. The measurement is repeated for slightly different values of the sample rotation $\theta$. In this way a two-dimensional $(\theta, \phi)$ distribution is obtained

\section{Experimental Details}

The experimental set-up is sketched in fig. 4. An electron beam with $40 \mathrm{keV}$ ( $0.2 \mathrm{~mm}$ diameter) impinges on the sample. The detector measures electrons emerging (within $0.1^{\circ}$ ) along a segment of the surface of a cone with half-cone angle of $44.3^{\circ}$. The measured segment is $\approx 11^{\circ}$ out of $360^{\circ}$ of the total cone. The incoming beam is along the axis of this cone. All electrons have thus been scattered over $135.7^{\circ}$. The incoming beam, sample surface normal and the trajectory hitting the center of the detector are all in the horizontal plane. The sample is mounted on a precision rotary feedthrough which can be rotated by a stepper motor. In this way the angle between the surface normal and trajectories of the detected electrons can be changed.

The beam current is measured (around 10nA) and the collected charge is integrated during the measurement. Data are acquired at each angle for a preset amount of charge. The electron analyser, consisting of a set of slit lenses followed by a hemispherical analyser, has a two-dimensional detector that measures the $\phi$-range and energy window of $40 \mathrm{eV}$ simultaneously. The energy resolution of the system is about $0.4 \mathrm{eV}$, the angular resolution in $\theta$ and $\phi$ is about $0.1^{\circ}$ [12. The intensity of the spectrum at each $\phi$ angle is determined for two different user-defined energy loss ranges. When the pre-set amount of charge was collected the $\theta$ angle was incremented and the measurement was repeated. Unless otherwise stated the $\theta$ - step size was $0.1^{\circ}$ used. A measurement sequence of 100 different $\theta$ angles takes about 24 hours.

The GaP crystals (supplied by MTI corp. $0.5 \mathrm{~mm}$ thick GaP wafers) were 


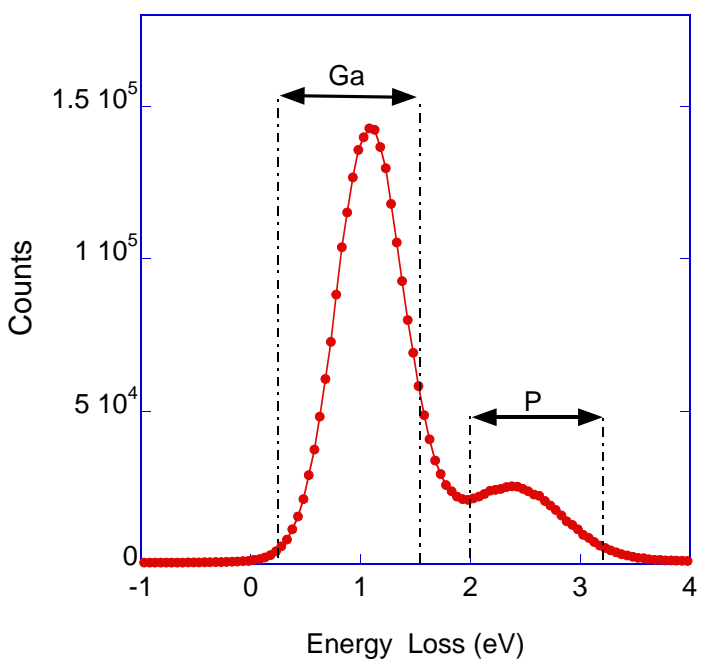

Figure 5: The quasi-elastic peak in the energy spectrum of electrons backscattered from GaP, consisting of partially resolved $\mathrm{Ga}$ and $\mathrm{P}$ components. The energy regions used to determine the $\mathrm{Ga}$ and $\mathrm{P}$ intensities are indicated as well.

sputtered with $2 \mathrm{keV} \mathrm{Ar}^{+}$ions and annealed at $\approx 600^{\circ}$ before the measurement, to ensure the presence of a well-ordered, clean surface. The orientation of the crystal was judged from the documentation of the supplier and the exact orientation was found by matching the observed intensity distribution to the calculated one. Most measurements were done using a wafer with a (0 01$)$ surface orientation, but (1 10$)$ wafers were used as well. Rotating the (l0 011$)$ crystal over $90^{\circ}$ along the surface normal, to access the point-group-symmetryinequivalent orientation of the crystal, could only be done by taking the sample out of the vacuum and rotating it manually in the holder. After reintroducing it into the vacuum the anneal treatment was repeated.

\section{Results}

For the measurement conditions in this study (scattering angle $\approx 135^{\circ}$, energy $40 \mathrm{keV}$ ), the recoil energy transferred to the target atoms in the large-angle deflection is well resolved 21. This makes it possible to distinguish electrons scattered from Ga from those scattered from $\mathrm{P}$ because the recoil energy scales as $1 / M$ with $M$ the mass of the scattering atom. This is illustrated in Fig. 5 showing the energy distribution of electrons quasi-elastically backscattered from GaP. Here we integrated over all detector $(\phi)$ angles. The statistics at a specific detector angle is about $100 \times$ less. Because there is some overlap between the $\mathrm{Ga}$ and $\mathrm{P}$ peaks, the energy loss regions used to determine the $\mathrm{Ga}$ and $\mathrm{P}$ peak intensities were chosen as indicated in the figure, excluding the region of significant overlap. The intensity of the Ga peak is considerably larger than that of the $\mathrm{P}$ peak, as the cross section scales in first approximation as $Z^{2}$, with $Z$ the atomic number. The separation of $\mathrm{Ga}$ and $\mathrm{P}$ is in good agreement with expectations for such a system. 
The $(001)$ crystal was oriented with a $\langle 110\rangle$ direction vertical. This means that a $\{110\}$ plane is approximately horizontal and always pointing towards the detector, and hence a $\{110\}$ Kikuchi band is always observed. When comparing the results with the calculations shown in Fig. 2 where these planes are seen at $45^{\circ}$, we can rotate the calculated intensity either over $\pm 45^{\circ}$ so a (110)-type plane is horizontal. As discussed before, these orientations are not equivalent and correspond to the difference between (110) and (110) in the crystal structure which we aim to discriminate with our measurements. Initially, the appropriate rotation was judged from by comparing the experiment with both options. Once the polarity was determined from the first measurement, all subsequent measurements confirmed this choice.

The first measurements we discuss are taken near $\langle 112\rangle$ zone axes, indicated as yellow and pink boxes in fig. 3a. Here the $\{110\}$ plane crosses the polar $\{111\}$ planes and we expect this Kikuchi band region to be sensitive to the crystal polarity. The results, for both polar orientations are shown in Fig. 6 and compared to calculations.

For easy comparison the calculations extends over a somewhat larger area than the measurement. The horizontal $(\theta)$ axis corresponds to directions from $25^{\circ}$ to $42^{\circ}$ between the outgoing trajectory and the surface normal. The $\phi$ direction extended from $-10^{\circ}$ to $6^{\circ}$ below and above the $\{110\}$ plane. The calculated patterns show a plethora of structures, the main ones are easily identified in the experiment as well. The $\langle 121\rangle$ zone axes stands out clearly.

The experimental patterns show less contrast, but the main features of the theory are easily identified. The actual measurement range is indicated by the red box drawn in the calculated distribution. As consistency requires, the measurement range is the same for the $\mathrm{P}$ and Ga distribution.

There are additional features in the experiment causing additional horizontal and vertical streaks, not seen in the theory. The horizontal lines relate to the detector response, e.g. inhomogeneities in the channel plate detection efficiency. The vertical lines seen sometimes in the experiment, but missing in the theory are attributed to channeling effects of the incoming beam as was demonstrated for silicon crystals elsewhere [14]. Because the incoming electrons are diffracted in a very similar way as the outgoing electrons, the probability that the incoming beam backscatters from a target atom changes when the crystal is rotated over $\theta$. Similar to different outgoing diffraction effects for $\mathrm{Ga}$ and $\mathrm{P}$, the incident beam changes are not always the same for $\mathrm{Ga}$ and $\mathrm{P}$. If incident beam diffraction happens it will affect the measurement at all outgoing $\phi$ values in the same way, causing vertical streaks in the outgoing $\mathrm{Ga}$ and/or $\mathrm{P}$ distributions.

To highlight the effect of the different polarity we take the ratio of the Ga and $\mathrm{P}$ signals in the lower panels. This has the additional advantage that detector inhomogeneities are mostly canceled out. In both experiment and theory, the horizontal bands ( $\{110\}$ planes) disappear if one takes the ratio. The other bands ( vertical $\{111\}$ planes planes and $\{311\}$ planes) remain visible. The vertical dashed-dotted line corresponds for the calculated distribution to $35.2^{\circ}$, the direction of the $\langle 121\rangle$ zone axis relative to the surface normal.

Clearly the calculated maximum intensity along the $\{111\}$ plane is either 


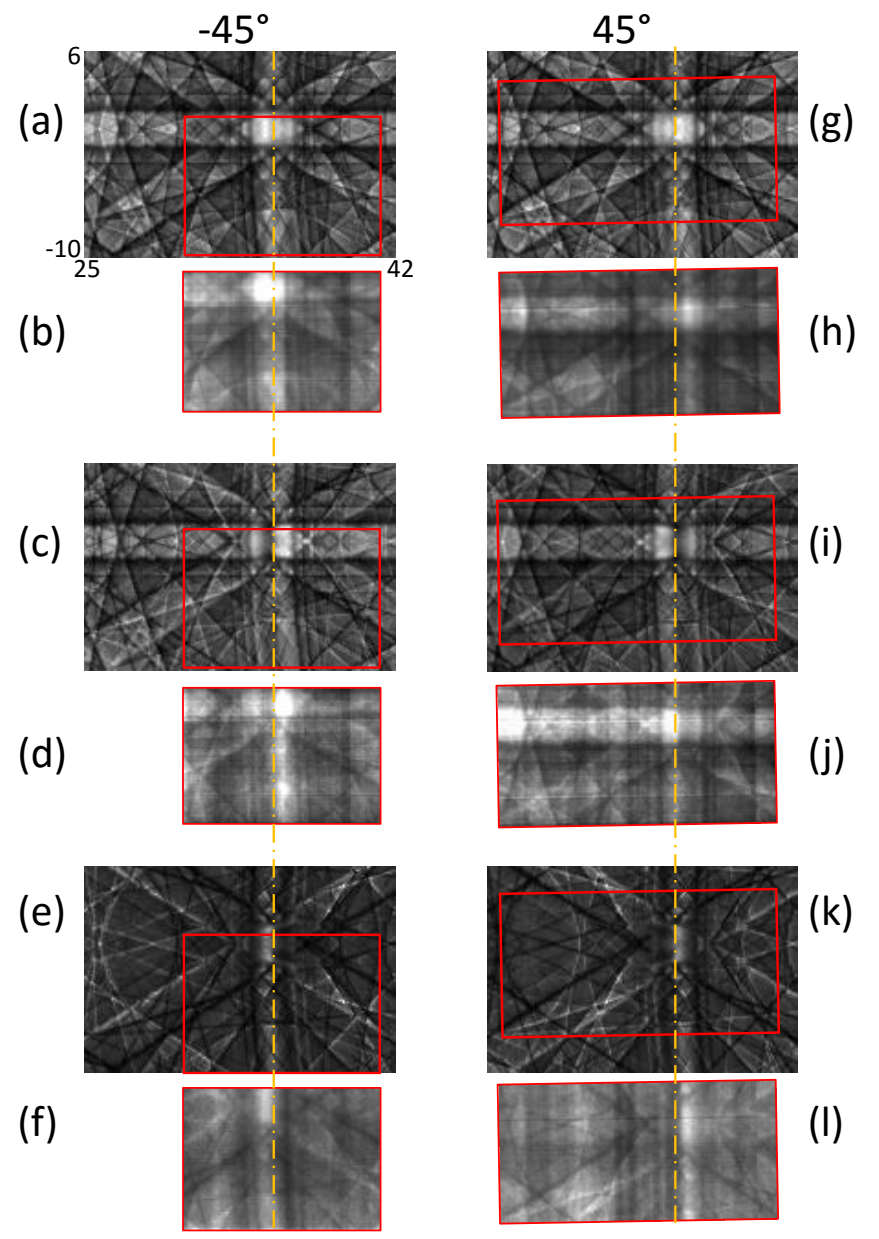

Figure 6: (a): Calculated Kikuchi pattern for electrons scattered from Ga near the [1 112$]$ zone axis. (b): Measured pattern for electrons scattered from Ga. The red box in (a) correspond to the area covered by the experiment. (c): The calculated pattern for electrons scattered from P. (d): The measured pattern for electrons scattered from P. (e): The calculated Ga intensity divided by the calculated $\mathrm{P}$ intensity. (f) The same for the measured intensity. (g-l): The same as (a-f) but now the crystal was rotated by $90^{\circ}$ around the [0 01 1] surface normal. Notice that (k) and (l) resemble the negative of (e) and (f). The yellow line marks the $\theta$ value of the $\left[\begin{array}{lll}1 & 1 & 2\end{array}\right]$ zone axis.

on the inside or on the outside of the dashed-dotted line. If Ga has its maximum on the inside, then $\mathrm{P}$ has its maximum on the outside and vice versa. In the experiment, the maxima of the $\mathrm{P}$ and Ga intensity are also clearly at different $\theta$ values. There is thus only one assignment one can make to the polarity that allows for a satisfactory comparison of theory and experiment, and the results are plotted accordingly.

There is, however, much more structure in the ratio distribution in both the experiment and measurement. They tend to have different 'polarity'. Lines that show up as white for the $-45^{\circ}$ orientation, appear as black at the $45^{\circ}$ 

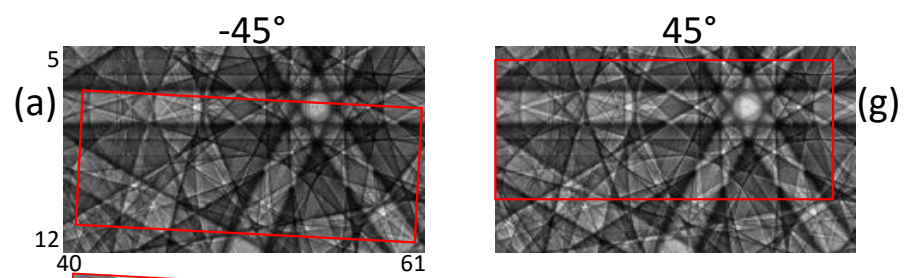

(b)
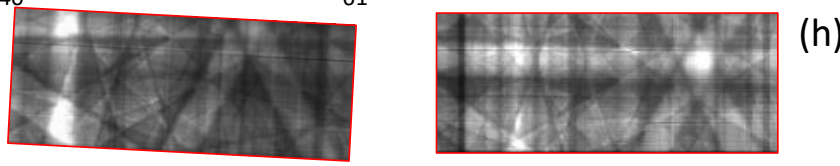

(c)
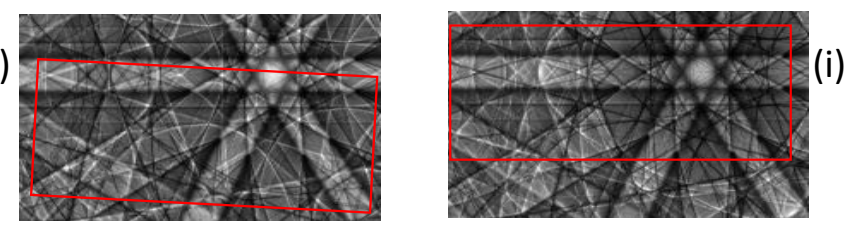

(d)
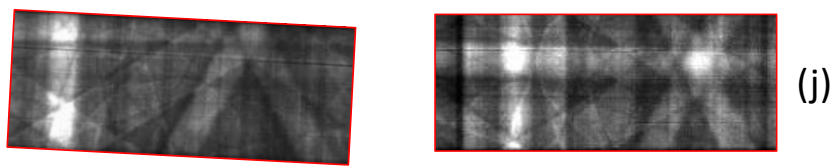

(e)
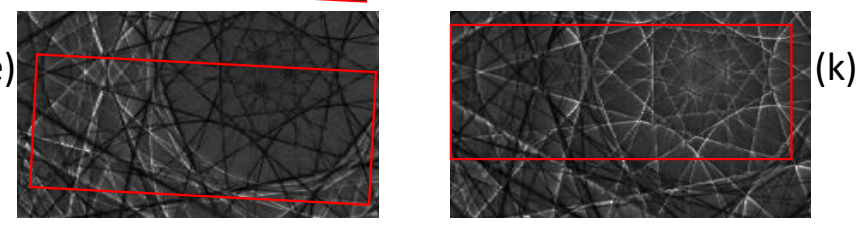

(f)
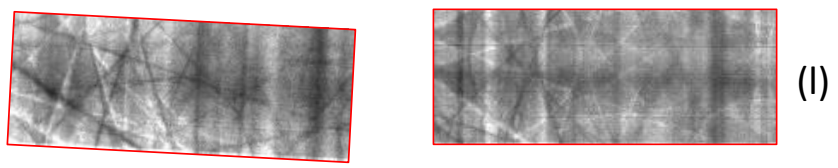

Figure 7: Same as Fig. 6 but now for directions near $\langle 111\rangle$ zone axes.

orientation. Inspection of the experiment show that many of these features are present in the measurement as well. Indeed for this choice of polarity the black lines in the experiment correspond to black lines in the calculation, and the same for the white lines.

In the calculations the origin of the white and black lines in the ratio distribution can be traced back to differences in the Ga and P intensity distributions themselves. To show this effect more clearly in the experiment, even longer measurement times would be required, as the contrast of the features is reduced.

Next we shift our attention to a similar measurement through the $\langle 111\rangle$ zone axis. These measurements correspond to the red and blue box in Fig. 3a. and are shown in Fig. 7. In this case there is a strong enhancement along a vertical band for an outgoing direction of $\theta=44.3^{\circ}$. This is when the incoming beam is in the close vicinity of the $\langle 100\rangle$ zone axis, where channeling effects leads to a pronounced enhancement of the backscattering probability [14]. The 
$\langle 111\rangle$ zone axis is visible near $\theta=54.6^{\circ}$, but when taking the Ga to $\mathrm{P}$ ratio the bands seen crossing here disappear. Again in the ratio plot dark and bright lines appear and the where dark lines appear for the $-45^{\circ}$ measurement light measurements appear for $45^{\circ}$ and vice versa. Near the $\langle 111\rangle$ direction there are exclusively dark lines or white lines. These tendencies are all reproduced by the experiment.

In Fig. 8, we show similar distributions near the surface normal $\left(\left\langle\begin{array}{lll}0 & 0 & 1\rangle\end{array}\right.\right.$ zone axis) corresponding approximately to the golden box in Fig. 3a. Here the measurement was done for only one of the two geometries $\left(-45^{\circ}\right)$. In the neighborhood of the star one can clearly see a white circle segment, which is only present for the $-45^{\circ}$ geometry. There are other dark and white lines visible in both the ratio plot of the experiment and theory, and again all the observed dark/white lines agree with the theory at $-45^{\circ}$, but disagree with the theory at $+45^{\circ}$. So even in rather close proximity to the $\langle 001\rangle$ zone axis there is an influence of the point group on the EBSD pattern (exactly at the zone axis, symmetry requires the absence of an effect). Note that in this case, when we take the $\mathrm{Ga}$ to $\mathrm{P}$ ratio, only the $\{110\}$ planes disappear, the other ones remain although the $\left\{\begin{array}{lll}0 & 0\end{array}\right\}$ planes appear to get narrower. This narrowing is a consequence of the fact that the $\mathrm{Ga}$ and $\mathrm{P}$ atoms here do not share the same plane (see Fig. 1), as explained elsewhere [18].

Finally, in Fig. 9 we show the intensity distribution near the [1 10$]$ direction, both as measured and as calculated, for Ga as well as for P. This measurement was done using a crystal with a [110] surface normal and the area measured corresponds roughly to the green box in Fig. 3a. In the theory, P shows a clear enhancement near this direction (again not exactly in the zone axis direction but at slightly positive $\phi$ values). For Ga this is not the case. The experiment shows also a clear maximum for $\mathrm{P}$ near the zone axis direction, but indeed no clear peak is seen for Ga.

Calculations show that the absence of an enhancement for Ga along the [110] direction is not specific for measurements $40 \mathrm{keV}$ as it was observed for calculations over a range of energies, down to $10 \mathrm{keV}$. Near the [110] zone axis direction the diffraction has to be described as a many beam case due to very pronounced dynamical scattering effects. The standing wave patterns of the angle-dependent exit probability that form then can have a complicated spatial distribution. Along the [110] direction there are strings of only Ga atoms and only $\mathrm{P}$ atoms. As we can see in Fig. 2 and Fig. 3b, along the low index zone axes $\langle 100\rangle$ and $\langle 110\rangle$, the $\mathrm{Ga} / \mathrm{P}$ ratio is significantly reduced. Along these directions, the $\mathrm{Ga} / \mathrm{P}$ ratio is dropping considerably below the theoretical value of 4.3 for the ratio of their Rutherford scattering cross sections, which indicates strong dynamical scattering effects along the atomic columns.

\section{Discussion}

The results presented in the previous section allow us to unambiguously assign the $\mathrm{GaP}$ crystal orientation with the resolution of the point group $\overline{4} 3 \mathrm{~m}$. 

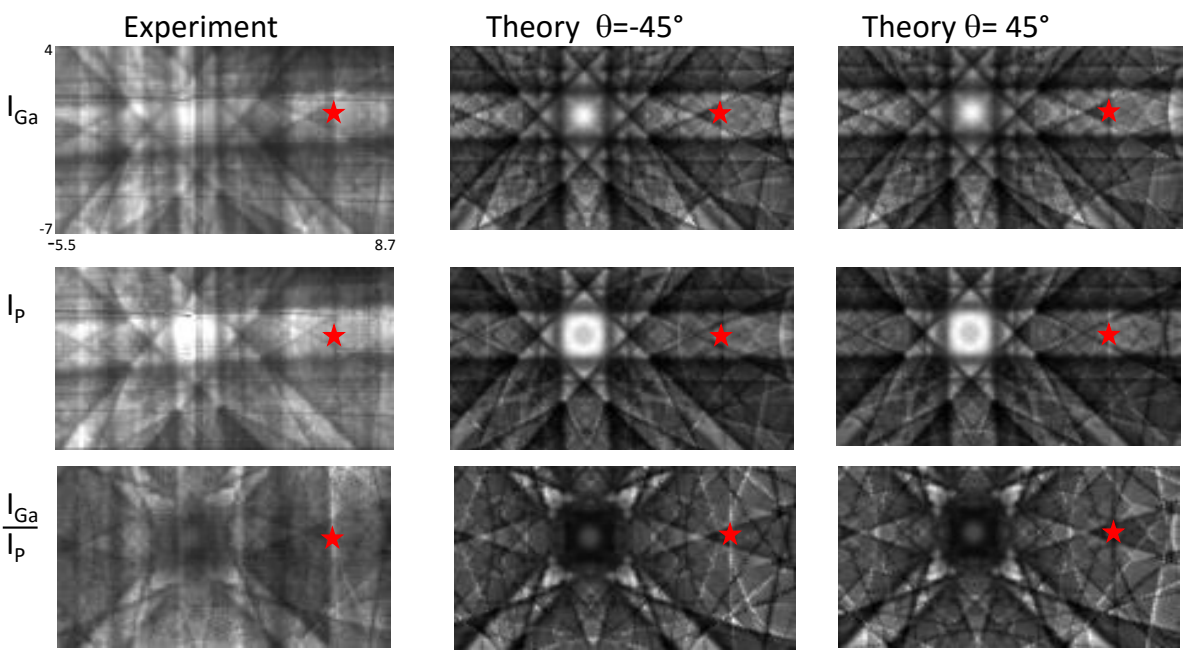

Figure 8: Measured intensity distribution near the [100] zone axis for Ga and $\mathrm{P}$ and the ratio of the $\mathrm{Ga}$ to $\mathrm{P}$ intensity (left column). A (110) plane is horizontal, easily visible in the intensity distribution of $\mathrm{Ga}$ and $\mathrm{P}$, but completely absent in the ratio. Due to the lack of 4-fold symmetry there are two non-equivalent crystal orientations possible. The calculated intensity distribution for both possibilities are given (center and right column). An example of a feature that differs is marked by a red star (a bow, most easily visible in the ratio plots). The experiment correspond to $\theta=-45^{\circ}$ orientation.

To be specific, in Fig 10 we show the two real space crystal orientations corresponding to a measurement of a $\langle 111\rangle$ zone axis of the cubic point lattice. Due to the lack of inversion symmetry that is caused by the different chemical occupation of the two $f c c$ sub-lattices of the zincblende structure, the [111] direction facing the analyzer is physically different from the $[\overline{1} 11]$ direction.

Our measurements presented above show explicitly that we can assign the experimental " $+45^{\circ}$ " orientation of the sample to the situation when the [1 111$]$ (or $[\overline{1} \overline{1} 1]$ ) direction is facing the analyzer in the (110) Kikuchi band. In this case (seen from the analyzer) the $\mathrm{P}$ atom is just before the nearest-neighbor $\mathrm{Ga}$ atom.

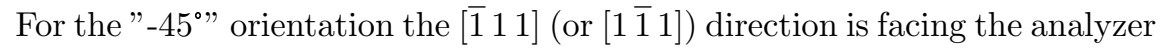
in the (110) Kikuchi band and the $\mathrm{P}$ atom is just behind the nearest-neighbor $\mathrm{Ga}$ atom. We see white lines in the $\mathrm{Ga} / \mathrm{P}$ ratio plot for the ' $+45^{\circ}$ ' orientation near a [1 111$]$ direction if $\mathrm{P}$ is just before Ga.

To obtain more insight into the mechanisms involved, it is helpful to plot the calculated intensity of $\mathrm{Ga}, \mathrm{P}$ and the sum for the two non-equivalent sample orientations $\left(+45^{\circ}\right.$ and $\left.-45^{\circ}\right)$. This is done in fig. 11 for a a segment along the $\{110\}$ bands that contains both $\langle 112\rangle$ and $\langle 111\rangle$ zone axes. The Ga distributions are about a factor of 4 more intense than the $\mathrm{P}$ distributions. This is because the simulations assume that the probability of large angle deflections scales as the Rutherford cross section i.e. like $Z^{2}$.

The difference of the two Kikuchi patterns for the two orientation are quite obvious, especially near the $\langle 112\rangle$ direction. This is even more obvious if we 

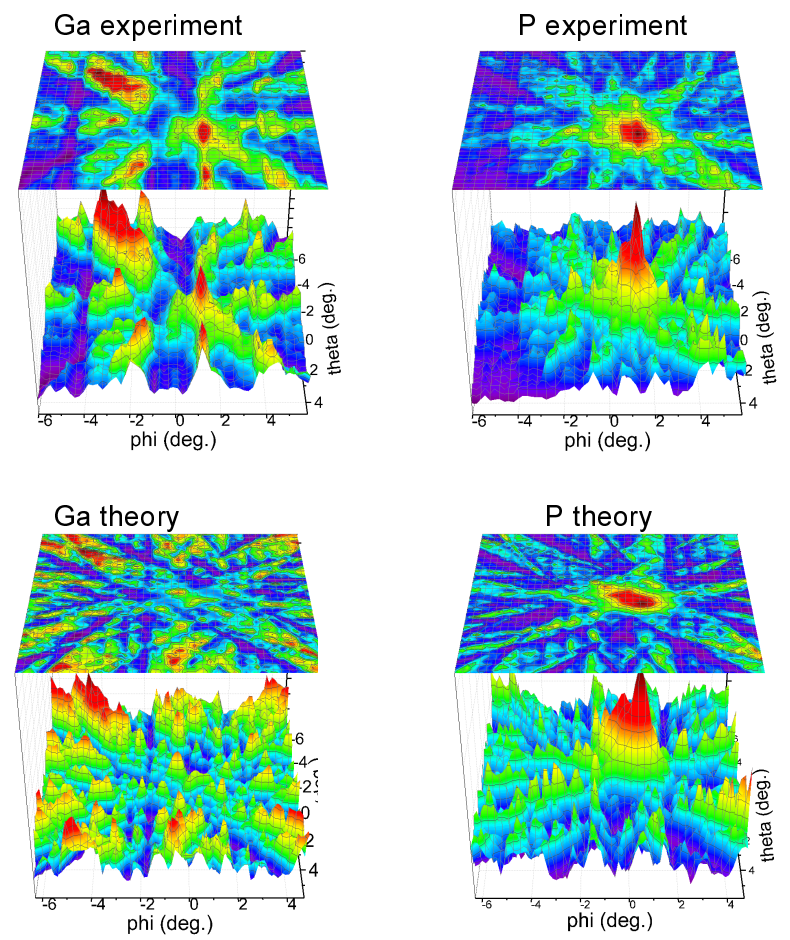

Figure 9: The intensity distribution for Ga and P near the $\left[\begin{array}{lll}1 & 1 & 0\end{array}\right]$ zone axis for Ga and P, both in the experiment and as calculated. Notice the absence of a noticeable enhancement for $\mathrm{Ga}$ in both theory and experiment for the [1 10$]$ zone axis.

plot the difference $I\left(45^{\circ}\right)-I\left(-45^{\circ}\right)$. For Ga the maximum difference is about $30 \%$ of the maximum intensity of $\mathrm{Ga}$, but the average difference is of course much smaller. The $\mathrm{P}$ difference pattern is a factor of 2 less intense as the Ga difference pattern whereas the $\mathrm{P}$ signal is 4 times less intense. In relative terms the $\mathrm{P}$ signal is thus more sensitive for the rotation over $90^{\circ}$.

Note that features appearing positive in the Ga difference pattern are negative in the $\mathrm{P}$ difference pattern. In the $\mathrm{Ga}+\mathrm{P}$ difference pattern, shown as well there is thus a partial cancellation and the magnitude of the difference as a fraction of the total intensity becomes even less.

The difference patterns for $\mathrm{Ga}$ and $\mathrm{P}$ have to a very large extent the same overall structure, up to a different sign. The difference between the two sample orientations is that the $\mathrm{P}$ and $\mathrm{Ga}$ atoms interchange their lattice position. So the movement from the $\mathrm{P}$ atom when going from $+45^{\circ}$ and $-45^{\circ}$ is opposite to that of Ga. Hence the fact that their difference distributions resemble each other, but have different polarities is plausible.

It is of interest to try to understand why the anisotropy for P, expressed as a fraction of the total $\mathrm{P}$ signal is twice as big as for Ga. For this it is helpful to consider the atomic differential scattering cross sections (DCS) of these atoms, as is done in fig. 12. The scattering processes that give rise to Kikuchi band formation are (coherent) small angle deflections. Here the differential cross 


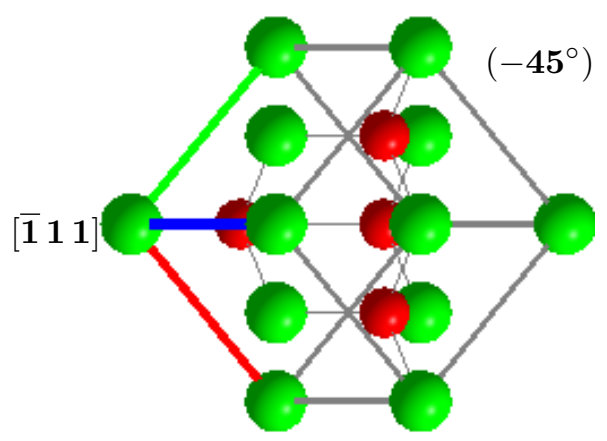

(a)

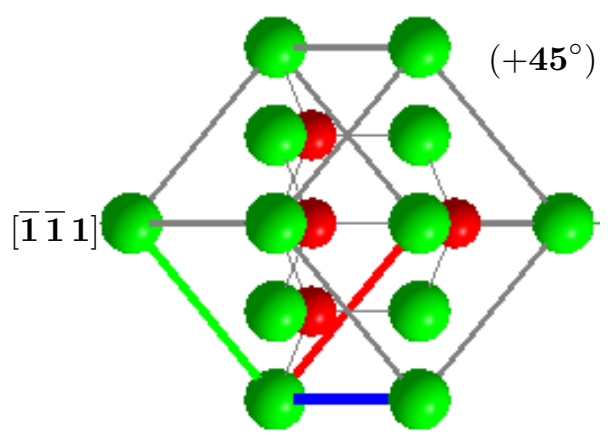

(b)

Figure 10: Real-space orientation of the GaP crystal corresponding to the $\pm 45^{\circ}$ measurements, green: Ga, red: P. The electron energy analyzer direction is to the left. Note how the two orientations are related by a $90^{\circ}$ rotation around [ $\left[\begin{array}{lll}0 & 0 & 1\end{array}\right]$ and effectively correspond to a different relative placement of $\mathrm{Ga}$ and $\mathrm{P}$ planes. (a) $-45^{\circ}$ corresponding to [1 11 ] (or [1 $\left.\overline{1} 1\right]$ ) facing the analyzer (b) $+45^{\circ}$ corresponding to [ $[\overline{1} \overline{1} 1]$ (or [1 11$]$ ) facing the analyzer

section for $\mathrm{P}$ is about half of that of Ga. For electrons back scattered from $\mathrm{P}$ rotating the crystal from $-45^{\circ}$ to $45^{\circ}$ corresponds to a change in relative position of the Ga atoms. For electrons back scattered from Ga this rotation corresponds to a change in relative position of the $\mathrm{P}$ atoms. For electrons incoherently backscattered from $\mathrm{P}$ the atom that changes position in both orientation ( $\mathrm{Ga}$ ) has the larger cross section and hence, the effect of the rotation is larger for electron backscattered from $\mathrm{P}$.

In the total $(\mathrm{Ga}+\mathrm{P})$ intensity there is still some effect of the rotation left, although, as a fraction of the total signal, is considerable less. For large angle scattering events the DCS of Ga is four to five times larger than the DCS of $\mathrm{P}$ (see Fig. 12). Thus the total intensity is dominated by that of Ga and the corresponding $\mathrm{Ga}$ anisotropy is only partially canceled by the $\mathrm{P}$ contribution, in spite of the fact that the $\mathrm{P}$ contribution has the relative larger anisotropy.

In the experiment we do not explicitly compare intensities after $90^{\circ}$ rotation, since this is hard to carry out with the required precision $\left(0.1^{\circ}\right)$ in our setup. 


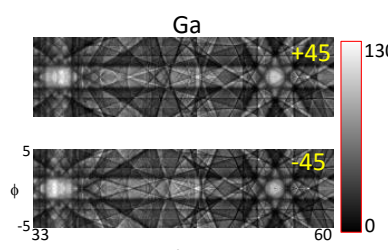

$\theta$

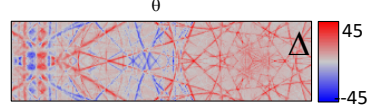

$\int_{\text {Calculated Intensity }}^{10-G a(+45)}$

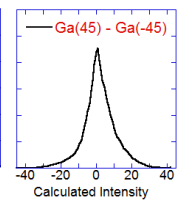

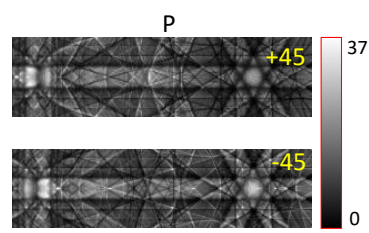
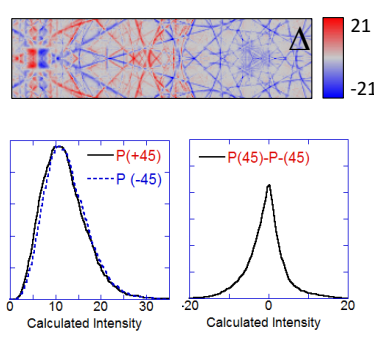
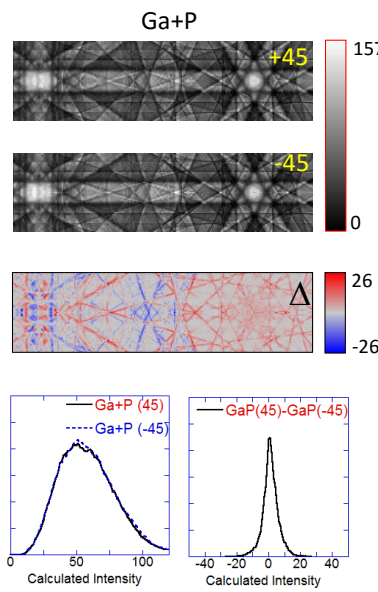

Figure 11: The top, left panel shows the calculated intensity for electrons scattered from Ga along the (110)-type plane, as reached rotating over +45 from the $(100)$ plane. and after rotating over $-45^{\circ}$. The panel marked $\Delta$ shows the difference in intensity of both orientation. The two panels at the lower left show the frequency distribution that a certain intensity occurred in either the calculated intensity for scattering from $\mathrm{Ga}$ and in the difference distribution. The central and right column show the same quantities for scattering from $\mathrm{P}$ and for combined scattering from $\mathrm{Ga}$ and $\mathrm{P}$.

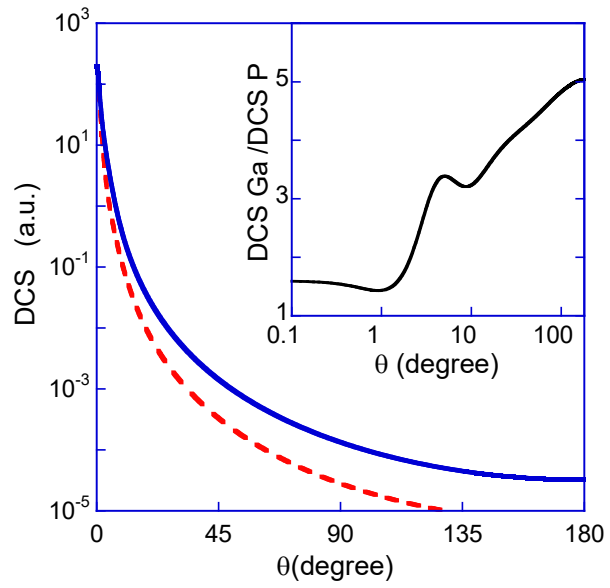

Figure 12: The differential cross section for elastic scattering (as calculated using ELSEPA 22]) from Ga (full line) and $\mathrm{P}$ (dashed line). The insert shows the ratio of both cross sections. 
Instead, the Ga:P intensity ratio provides a much more direct and reliable signal. The $\mathrm{Ga} / \mathrm{P}$ ratio amplifies the effect, as an increased Ga intensity implies a reduced $\mathrm{P}$ intensity, and vice versa. It is also obvious that, given the behavior of $\mathrm{Ga}$ and $\mathrm{P}$ separately, the obtained intensity ratio changes 'polarity' if the crystal is rotated by $90^{\circ}$. Thus, determining the Ga/P intensity ratio in elementselective Kikuchi patterns is an elegant way to study the effect of the absence if inversion geometry on these Kikuchi patterns.

\section{Conclusion}

Using the example of absolute point-group resolved orientation determination of GaP, we demonstrated that angle-resolved ERBS measurements using an electrostatic electron energy analyser can provide unique access to chemically resolved crystallographic information. In comparison to element-integrated Kikuchi pattern measurements like in the method of electron backscatter diffraction [9, 10, the effect of the absence of a proper 4-fold rotation axis in the point group of $\mathrm{GaP}$ can be sensed with a much higher visibility via the elementresolved $\mathrm{Ga}$ to $\mathrm{P}$ intensity ratio. In particular, it was demonstrated that the asymmetry recently observed in conventional Kikuchi pattern measurements for the (111) plane is present for both Ga and P, and that it is of opposite nature. The remaining asymmetry seen for the total Kikuchi pattern is due to the larger contribution of electrons scattered from Ga compared to P. Besides the asymmetry of the (111) plane there were many more features seen in the Ga/P intensity ratio which are caused by the lack of an inversion center. In particular, near the [1 11 ] zone axis these features all favor the Ga intensity over the P intensity, then, when the crystal is rotated over $90^{\circ}$ the opposite is true. Moreover it was confirmed experimentally that the Ga atoms do not contribute to the increased intensity along the [110] directions, in agreement to the calculations.

In short, the experiments shown here illustrate a possible way of studying the effects of diffraction on the chemical sub-lattices in non-centrosymmetric crystals. In particular it is possible to determine the 'polarity' of the crystals. This is an important issue for many technological cases, mentioned in the introduction, which in most cases (when 'Friedel's law' applies) can not be addressed by X-ray diffraction. The method demonstrated here uses even higher energies than is possible in X-ray photoelectron diffraction experiments [17, and the electron beam potentially can provide local resolution on sub-micron scales,

thus facilitating the local determination of the polarity of thin epitaxial layers.

\section{Acknowledgements}

This work was made possible by a grant of the Australian Research Council.

[1] Carmelo Giacovazzo, Hugo Luis Monaco, Gilberto Artioli, Davide Viterbo, Marco Milanesio, Gastone Gilli, Paola Gilli, Giuseppe Zanotti, Giovanni Ferraris, and Michele Catti. Fundamentals of Crystallography. IUCr Texts 
on Crystallography, Vol. 7. Oxford University Press, Oxford, 2011. ISBN 9780199573653. doi: 10.1093/acprof:oso/9780199573653.001.0001.

[2] Kenji Morizane. Antiphase domain structures in GaP and GaAs epitaxial layers grown on Si and Ge. Journal of Crystal Growth, 38(2):249 - 254, 1977. ISSN 0022-0248. doi: 10.1016/0022-0248(77)90305-0.

[3] L. T. Romano, J. E. Northrup, and M. A. O'Keefe. Inversion domains in GaN grown on sapphire. Appl. Phys. Lett., 69(16):2394-2396, 1996. doi: $10.1063 / 1.117648$.

[4] Fude Liu, Ramon Collazo, Seiji Mita, Zlatko Sitar, Stephen J. Pennycook, and Gerd Duscher. Direct Observation of Inversion Domain Boundaries of GaN on c-Sapphire at Sub-Angstrom Resolution. Advanced Materials, 20 (11):2162-2165, 2008. ISSN 1521-4095. doi: 10.1002/adma.200702522.

[5] Kerstin Volz, Andreas Beyer, Wiebke Witte, Jens Ohlmann, Igor Nemeth, Bernardette Kunert, and Wolfgang Stolz. GaP-nucleation on exact Si (001) substrates for III/V device integration. J. Cryst. Growth, 315(1):37 - 47, 2011. ISSN 0022-0248. doi: 10.1016/j.jcrysgro.2010.10.036.

[6] S. Liebich, M. Zimprich, A. Beyer, C. Lange, D. J. Franzbach, S. Chatterjee, N. Hossain, S. J. Sweeney, K. Volz, B. Kunert, and W. Stolz. Laser operation of $\mathrm{Ga}$ (NAsP) lattice-matched to (001) silicon substrate. Appl. Phys. Lett., 99(7):071109, 2011. doi: 10.1063/1.3624927.

[7] Ricarda Maria Kemper, Thorsten Schupp, Maik Häberlen, Thomas Niendorf, Hans-Jürgen Maier, Anja Dempewolf, Frank Bertram, Jrgen Christen, Ronny Kirste, Axel Hoffmann, Jrg Lindner, and Donat Josef As. Antiphase domains in cubic GaN. J. Appl. Phys., 110(12):123512, 2011. doi: $10.1063 / 1.3666050$.

[8] A. Beyer, B. Haas, K. I. Gries, K. Werner, M. Luysberg, W. Stolz, and K. Volz. Atomic structure of (110) anti-phase boundaries in $\mathrm{GaP}$ on $\mathrm{Si}(001)$. Appl. Phys. Lett., 103(3):032107, 2013. doi: 10.1063/1.4815985.

[9] Aimo Winkelmann and Gert Nolze. Point-group sensitive orientation mapping of non-centrosymmetric crystals. Applied Physics Letters, 106:072101, 2015. ISSN 1077-3118. doi: 10.1063/1.4907938.

[10] G. Nolze, C. Grosse, and A. Winkelmann. Kikuchi pattern analysis of noncentrosymmetric crystals. J Appl Cryst, 48:1405, 2015. doi: 10.1107/ s1600576715014016.

[11] Matthew J. Burch, Chris M. Fancher, Srikanth Patala, Marc De Graef, and Elizabeth C. Dickey. Mapping $180^{\circ}$ polar domains using electron backscatter diffraction and dynamical scattering simulations. Ultramicroscopy, in press, 2016. doi: 10.1016/j.ultramic.2016.11.013. 
[12] M. Vos, G. P. Cornish, and E. Weigold. A high-energy (e,2e) spectrometer for the study of the spectral momentum density of materials. Rev. Sci. Instrum., 71:3831-3840, 2000. doi: 10.1063/1.1290507.

[13] M. Vos, M.R. Went, and A. Winkelmann. Quantitative measurements of Kikuchi bands in diffraction patterns of backscattered electrons using an electrostatic analyzer. Ultramicroscopy, 109:1211, 2009. doi: 10.1016/j. ultramic.2009.05.004.

[14] M. Vos and A. Winkelmann. Two-dimensional kikuchi patterns as measured using an electrostatic analyser. Ultramicroscopy, 171:19, 2016. doi: 10. 1016/j.ultramic.2016.08.015.

[15] C.S. Fadley. The study of surface structures by photoelectron diffraction and Auger electron diffraction. Synchrotron Radiation Research, page 421, 1992.

[16] Scott A. Chambers. Epitaxial film crystallography by high-energy Auger and X-ray photoelectron diffraction. Advances in Physics, 40:357, 1991. ISSN 1460-6976. doi: 10.1080/00018739100101502.

[17] Jesse R. Williams, Igor Piš, Masaaki Kobata, Aimo Winkelmann, Tomohiro Matsushita, Yutaka Adachi, Naoki Ohashi, and Keisuke Kobayashi. Observation and simulation of hard X-ray photoelectron diffraction to determine polarity of polycrystalline zinc oxide films with rotation domains. J. Appl. Phys., 111:033525, 2012. doi: 10.1063/1.3682088.

[18] Aimo Winkelmann and Maarten Vos. Site-specific recoil diffraction of backscattered electrons in crystals. Phys. Rev. Lett., 106:085503, 2011. doi: 10.1103/PhysRevLett.106.085503.

[19] M. Vos, A. Winkelmann, and G. Nolze. Element-specific Kikuchi patterns of rutile. Ultramicroscopy, 156:50, 2015. doi: 10.1016/j.ultramic.2015.04.018.

[20] Aimo Winkelmann, Carol Trager-Cowan, Francis Sweeney, Austin P Day, and Peter Parbrook. Many-beam dynamical simulation of electron backscatter diffraction patterns. Ultramicroscopy, 107:414-21, 2007. ISSN 0304-3991. doi: 10.1016/j.ultramic.2006.10.006.

[21] M.R. Went and M. Vos. Investigation of binary compounds using electron Rutherford back scattering. Appl. Phys. Lett., 90:072104, 2007. doi: 10. $1063 / 1.2535986$.

[22] F. Salvat, A. Jablonski, and C. J. Powell. ELSEPA Dirac partial-wave calculation of elastic scattering of electrons and positrons by atoms, positive ions and molecules. Comput. Phys. Commun., 165:157-190, 2005. doi: 10.1016/j.cpc.2004.09.006. 